\title{
A Brief Insight on Simulation and Space-Time
}

\author{
Reyan Kumar Sapkota
}

\begin{abstract}
Throughout most of human history, events and phenomena of interest have been characterized using space and time as their major characteristic dimensions, in either absolute or relative conceptualizations. Space-Time analysis seeks to understand when and where (and sometimes why) things occur. Ever since Einstein introduced this topic in his "General Theory of Relativity" (a remarkable feat) in 1915, many explanations, assumptions about Space-Time have been published. The authentic archives of Space-Time have helped us to predict and express the ongoing spatial phenomena of the Universe. The strangeness of Space-Time forces Young Researchers and Physicists to study upon the current predictions and theories, sink into the ocean of Space-Time mystery and come up with their own predictions. Besides Space-Time, the predictions about us being in a Simulation is a recent concept. Nick Bostrom's trilemma "the Simulation argument", published in 2003 led to the commencement of further oddly satisfying, real life evidence enriched, research papers. "Two possibilities exist: either we are alone in the Universe or we are not. Both are equally terrifying." by Andrew C Clarke. Throughout the paper, the Loaf Space-Time model and brief insights on other hypotheses about the structure of the mysterious space-time will be presented with insightful examples, authentic research outcomes, which will be linked with the Simulation Hypothesis.

Keywords: Worldlines, General Relativity, Lorentz Transformation, Planck's Era, Inflation, Singularity, Matrix, Simulation, Continuum
\end{abstract}

\section{INTRODUCTION}

$\mathrm{S}_{\text {pace is where things happen and Time is when things }}$ happen. According to our literature and common sense, Where and When are analogous and related. This can be a simple statement for convincing a lay man about the presence of the Space-Time structure of the Universe. The concept of " Simulation" can be explained pretty well through the Space-Time Continuum.Michio Kaku, theoretical physicist in string theory, claimed that the expansion of the universe could be explained through the dimensional analysis of information, which he referred to as quantum energy, and how it creates space with respect to time. His concept was based on Albert Einstein's fourth dimension, called space-time, which serves as a tool for analyzing the physical phenomena of this startling Cosmos. In 1925, Werner Heisenberg proposed a hypothetical claim that without space and time, it is difficult to formulate a physical theory, leading to the foundational assertion of string theory, which is considered one of the most acceptable theories by scientific communities to qualify for the unifying fundamental nature of forces, for formulating "The Grand Unified Theory".Space-Time is a mathematical model where Space(3D) and Time (a dimension) is fused to form a single 4-Dimensional Continuum.

Manuscript received on 29 September 2021 | Revised Manuscript received on 06 October 2021 | Manuscript Accepted on 15 October 2021 | Manuscript published on 30 October 2021.

* Correspondence Author

Reyan Kumar Sapkota*, Department of Physics, St. Xavier's College, Maitighar, Kathmandu. Email: reyansapkota.108@gmail.com

(C) The Authors. Published by Lattice Science Publication (LSP). This is an open access article under the CC-BY-NC-ND license (http://creativecommons.org/licenses/by-nc-nd/4.0/)
Until the dawn of the $20^{\text {th }}$ century, the universe was thought to be composed of a spatial arrangement of 3 length coordinate systems. But Einstein brought in the concept of Space-Time Continuum to support his Theory of Relativity. Through this concept a conclusion about the invariant phenomenon of light's speed can be explained. Space-time diagrams are also very useful for describing the trajectories of material objects, especially when making quantitative statements about the proper time experienced by an observer.

The following paper tries to show that the Universe is a simulative existence embedded into the vastness of the Space-Time continuum. It tries to prove the argument through various mathematical and descriptive models.

\section{THEORY AND CALCULATION}

\subsection{Time Dilation}

Every Physics enthusiast must be accountable with the famous "The Twin Paradox". This paradox encompasses the concept of Time Dilation. The bewildered young Physicists' query about the reason for Time Dilation is the reason for this paper's initiation. Let's come to the point. Time Dilation is the difference between the time measured by two different clocks, either due to having their different relative velocities with respect to one another, or being in different gravitational fields or due to difference in trajectory of the motion of accelerated particles in the space-time continuum.

Value of specific trajectory of the particle in certain acceleration:

$$
\frac{1}{c} v(t)=\frac{2}{\sqrt{8}}(1-\lambda c t) ; 0<t<2 / \lambda c .
$$

Integrating the above equation, we obtain,

$$
T^{\prime}=\frac{(2+\pi)}{4 \sqrt{2}} \frac{2}{\lambda c} \approx 0.908914 \frac{2}{\lambda c}
$$

This shows that the time experienced by the accelerated object ( $T$ ') is less than the time experienced by the inertial observer. The accelerated object experiences that the time is moving very slowly compared to the inertial observer (observer at 0 acceleration). The faster it moves through space the slower it moves through time. This is what time dilation refers to.

Also, from Lorentz Transformation Scale:

$$
t^{\prime}=\frac{t}{\sqrt{1-\frac{v^{2}}{c^{2}}}}
$$

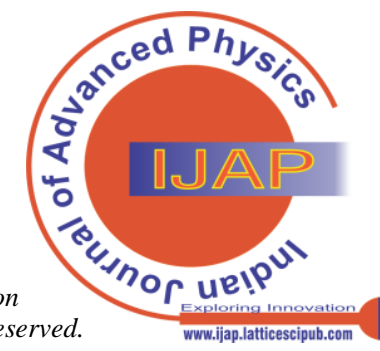




\subsection{Loaf-Stack Model}

Now, let's try to grasp this in another way. Einstein once considered space-time as a loaf of bread (Time Compression Theory). He said Space-time is like a loaf of bread where each slice is a different slice and each slice acts as a different freeze frame during the continuous notion of the ever-expanding Universe (at the speed of 72 kilometers per second per megaparsec). The whole loaf exists now, but we are observing our way through just one slice at a time, so it seems to us like the slices ahead of us do not exist yet, but they do exist in the loaf. We are currently in one of those very slices and are experiencing a Stubborn Illusion, so called Time (as mentioned by Einstein).

Now imagine yourself in the slice and also imagine an extraterrestrial creature, far about millions of light years in that slice. Consider that a creature moving towards the Earth has a difference in angle of 00 . Now consider the not orient with the time world line if yours. The line might be disoriented with even a very small angle. But this small angle difference will result in a large difference between the length of the worldlines between that of mine and the creature. So that creature's world line might be oriented towards my future or my past. And my world line might be oriented toward his past or future and vice versa. This difference in worldlines causes a difference in passage and flow of time for that creature and for me. This causes time dilation for me and that creature. So that creature can travel through my past or future if he follows that direction or orientation of his original worldline and similar case for me as well.

\subsection{Simulation}

Are we real or are we programmed to be real? Such questions question our existence and fate. Over the advancement of technologies, people began to think and question their existence. How is the Universe so strange? Why is everything happening for a purpose? Why do we Homo sapiens sapiens (so considered as the smartest living being to live on the Earth so far) are still hitting around the bushes while explaining about the realities of the Universe? Religion and myth say that Everything about our life is written by "The God". What if "The God" is a programmer who is simulating our entire existence and who controls our fate and fate of the Universe?

Computer Simulation is a reproduction of a behavior using computer software to create a dynamic environment under desired mathematical and physical programmable laws.

A version of the simulation hypothesis was first theorized as a part of a philosophical argument on the part of René Descartes, and later by Hans Moravec. The philosopher Nick Bostrom developed an expanded argument examining the probability of our reality being a simulation. Much evidence like "The Mandela Effect", "Sinbad Genie Movies", "The DNA-data theories" suggests some convincing evidence in the favor of we being Simulated by someone whose existence is beyond our comprehension and imagination.

Now, let's get into the real business. Now again imagine space-time as the loaf of bread as I have mentioned above. creature's orientation is such that his Time Worldline does

Let me add a few points on this version of Imagination and visualization.

Our past, present and future is already programmed in the slice of the space-time (n times the slice= the entire space-time (or think it as a loaf as a bread)). The present, past and the future may just be an illusion. In the day to day life we experience time as a continuous flow. But it can be pursued as the series of snapshots of each small moment of about Plank's unit (basic and fundamental entity for me. Nothing can get much smaller than this.) The time we are experiencing can be understood as the collection of the snapshots. And everything that happens can be visualized as the unfolding or flipping of these series of snapshots. And if we picturize and try to align each moment or snapshot lined up to form the very nature of the flow of time and existence of events with it, every moment of the Earth, Every moment of the Universe (ever since its birth to the formation of stars to the creation of the Earth, to the events occurring now (like an explosion of the star, or a meteor striking any planet, the Earth revolving around the Sun or whatever that is happening at the same time at the different corners and regions of the Universe) to death of the Universe(meaninglessness of time) into a single comprehendible structure by stacking or piling them laterally, then we can imagine closed loop as a shape as of the Loaf of bread or a 3D parabolic curve which is nothing but a SPACE-TIME. These events are programmed or set into the slice or the snapshots of the space- time. Time seems to go forward because these snapshots of the minute events are oriented forward. So, what is going to happen in the next 5 minutes is already set into that snapshot in that slice or in that snapshot. (in whatever way you comprehend).

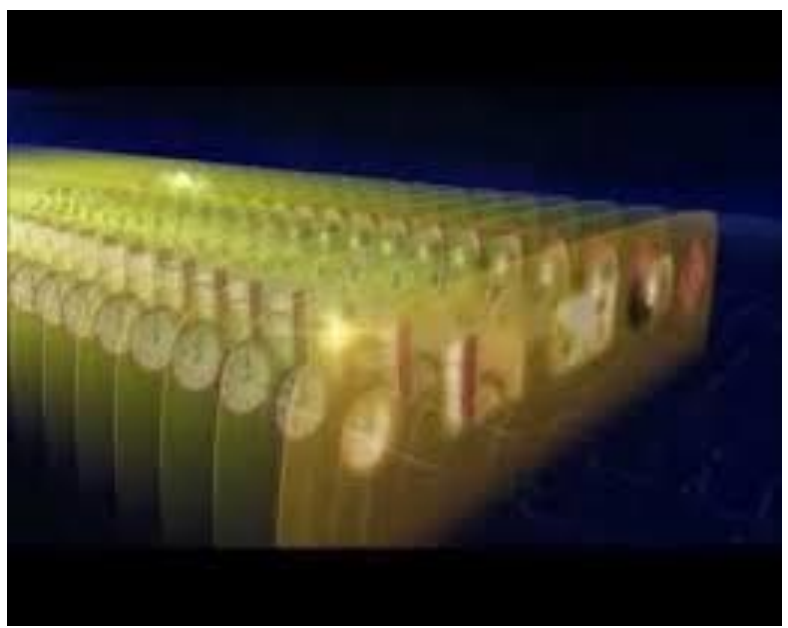

Fig: Loaf-Stack Model of Space-Time

Similarly, our future might already be set in those upcoming slices and snapshots, but for us those upcoming slices or those moments in the space-time continuum is impossible to reach (for now*). Same kind of concept holds true for the past as well. Each event is already set or programmed in that particular slice. Now let's imagine the Loaf of bread as a flip book and the slices as the paper(snapshots) of the flip book.

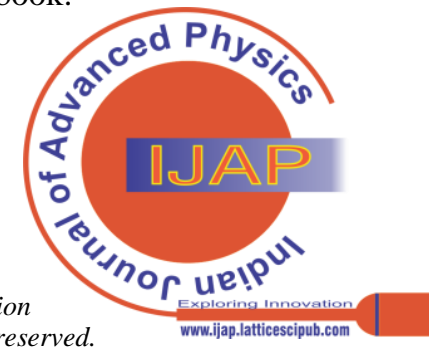


So, while flipping through the snapshots of the flip book, we feel as if the characters drawn in the pages of the flip book are moving in real time. The same case holds for the flow of time. Imagine the space-time of Loaf structure as a flip book where each page (with snapshots) are the parts or a simple phase or moment of that space-time. From the Origin of the Universe (Big-Bang (if this event actually happened)) to the end or death of the Universe (The point where Time becomes meaningless), every single bit of event is already set is these pages (as the snapshots) and the flow of time is just the moment of the flip book. Our future already exists. It is already programmed as bits of information.

This might seem absurd for many. But this is what I think is going on. That slice of the loaf or the page of the flipbook of the Space-time can be thought as a small bit of information storage system and our existence, activities and fate is the data being stored in that information storage system (i.e. the slice or the snapshot in the paper of the flip book, with whatever you may feel comfortable to grasp).

Every bit of basic unit of information [I would like to call it as a Planck Unit] is stored in the slice and the snapshot flows as if it is being animated or programmed to move forward in a particular direction and orientation. Within these slices the Laws of Classical Mechanics, including Newtonian Mechanics, upholds true and valid. Under the course of the events and flow of the snapshots, new law underlies. The flow of time, its existence and remorselessness, the events beyond this slice (slice where we Homo sapiens inhabit), other unexplored slices show different laws and phenomena. But one thing is sure: the entire functioning of our SPACE-TIME (concept explained above), the entire flow, the modulation and its loop and its discontinuation and so on is governed by a single unified law or I say it as a Principal Protocol or Master Algorithm. The maximum physical laws among the different slices might be different, but for some events among the slices, the laws might be related and also similar.

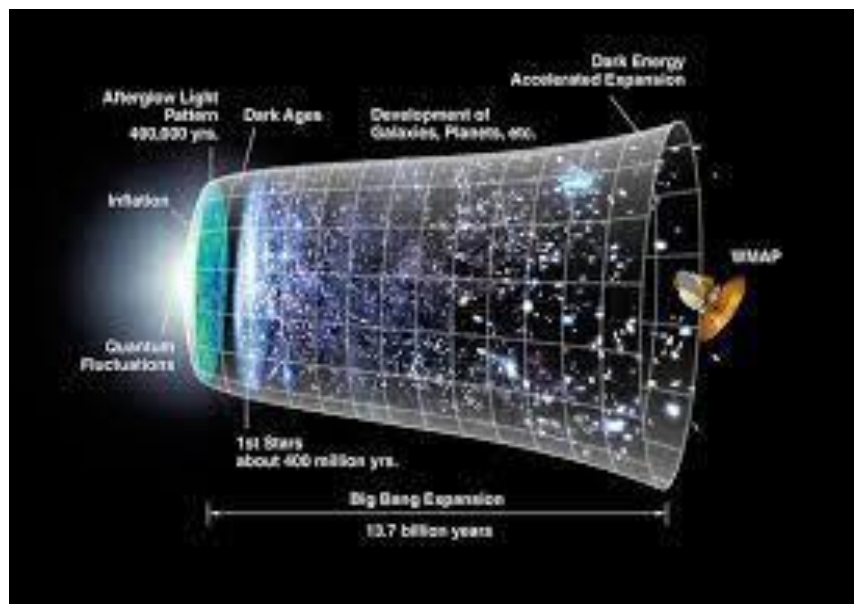

\section{RESULTS, EVIDENCE AND DISCUSSION}

The evidence that proves the Simulation argument is mentioned below. The following real life instances are the evidence and also the results that satisfy the aforementioned mathematical and descriptive theory.

\subsection{Deja Vu}

It is the most common example of this idea. We all have experienced, at least once in our life till date. When you see something or experience something deep inside your subconscious mind you begin to realize out of nowhere that you have already experienced this very moment sometime before at some particular time but in reality, you haven't experienced this moment anywhere before. This is Deja Vu.

To explain this, I would like you to close your eyes and imagine the two models that I have mentioned above about the structure of the Space-Time, the slices or snapshots model and the flip book model. Now combining these two models can think of SPACE-TIME as the slices aligned in a manner as that in flip books where it can undergo continuous illusionistic motion like that we observe while flipping a flip book containing a series of snapshots. Our existence can be pursued as the flow which we perceive during the course of flipping of the data or fate programmed on the snapshots or slices which are stacked as bundles and combined to form so called SPACE-TIME. We are already programmed to do something. Our origin and fate are already decided and so for the Universe.

\subsection{Bugs and Restrictions}

Now consider all these hypotheses and reflect upon them. Above views and ideas clearly try to show that we are in a Simulation. We are under Simulation governed by some supreme beings whom we cannot understand and comprehend because that's what we are programmed to be. We are set within certain limits. No one can raise a question upon this fact. Why is it so? It is because of the Simulation. One day the video games will be difficult to distinguish from reality. The same is happening with reality. Someone has programmed and designed our existence, fate and the entire information are stored in the slices or the snapshots of the SPACE-TIME. In fact, the SPACE-TIME serves as a hard drive of the data and information of the Fate of all the events which are undergoing in every part of the Universe, every unreachable and unimaginable and impossible points of the Universe where human endeavors cannot reach. This access to such areas of the Universe is limited by our potentials and this potential is already programmed. The codes written by some unknown being governs our potential, our entire activities and our fate. We and our Universe are bound by the set of rules and are governed accordingly. The data structuring, the coding, analysis, errors, bugs can be seen in the Universe now and then and it can be viewed in a convincing way in SPACE-TIME -slices platform. The bugs can be some events that break the laws of physics like Tachyons (the particle which was believed to be faster than light). Since light's speed's dominance is unbeatable at least in our observable Universe, we can say due to some bugs arising in the codes, Tachyon were rumored to be faster than light. And more interestingly Tachyons are hypothetical. They don't exist. How amazing!!! Some bugs over the Slices of space- time during the late 60s Tachyons' concept emerged as the particle that breaks the laws of nature. There are many such ideas in simulation theory which seem absurd but interesting, silly but logical.

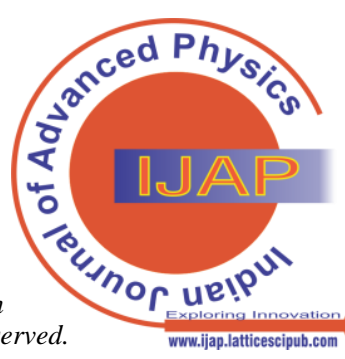


Another bug might be the Paranormal Activities. The scenarios that we experience sometimes like the unexplained sounds, sightings etc. can be the glitches of the Simulation world where we are living.

Some rare Savants see a pattern in the surroundings. When they were asked to draw the pattern, they observed in the surrounding, the patterns showed closed resemblances with the Simulation matrix patterns. The savants might be those special glitches which could observe the Matrix patterns of the Simulated Universe.



Mathematical pattern drawn by Savants.

\subsection{Velocity of Light}

Everybody knows nothing in the Universe is faster than light. Since for such a Simulation of the World to be carried out, a smooth, fast and powerful microprocessor sort of thing must exist. So, Light's velocity might be the speed of that microprocessor.

\subsection{Mathematics}

The Universe possess a single, irrevocable language i.e. Mathematics. The Laws of Mathematics do not hold untrue in any point of the Universe. The Universe is so Mathematical. This discloses the fact that Mathematics might be the platform in which our Universe is created. In simple words, Mathematics might be the programming language of the Universe. Everything in the Universe can be explained in a Mathematical language. Strings, Atoms, DNA and every other basic entity and advanced phenomena can be explained Mathematically without affecting its laws. In fact,

It was recently found that DNA is programmable. (It is a solid proof of the World being in a Matrix)

\subsection{Superstring Equations}

Jim Gates Jr says that he observed computer codes in the basic strings of Nature. The String resembles bits of Computer codes of ones and zeros. He even says it is a special kind of computer code discovered by Claude Shannon (the father of information theory). These codes were found deep in the Equations that explain String theory. Therefore, this also provides strong evidence on the Universe being a Matrix.

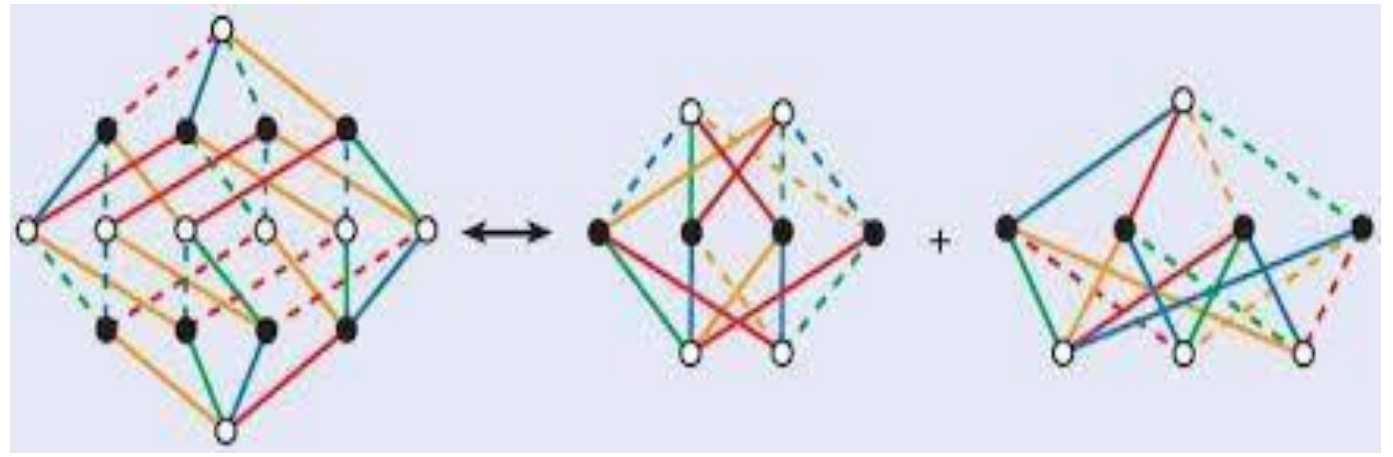

Fig: Computer Codes in Superstring Equation

\section{CONCLUSION}

The review article revitalizes the concept of the Simulated Universe into a new dimension of comprehension and evaluation. The various theories that support the Simulation theory seem undeniable. Research has been extensively carried out, but the public readers and appreciators haven't shown much relevance to the core of the topic. Insight into various pieces of evidence definitely arouses the curiosity among young researchers. This review article's effort to illustrate the motive of the Simulation theory as a legit argument have been commendable by various notable readers.

\section{ACKNOWLEDGEMENTS}

Gratitude pours from the bottom of my heart to those who have supported and helped review the article into a readable and referable manuscript. The guests, esteemed judges, fellow delegates and audiences of Young Scientists
Summit, Nepal deserve sincere gratitude for the creation of this manuscript. I also acknowledge the selfless guidance from Mr. Gopal Jha (Department of Physics, St. Xavier's College).

Finally, I thank all of those unmentioned ones who have supported me with research materials, time, guidance and motivation.

\section{REFERENCES}

1. World Lines. (n.d.). [ebook] Santa Barbora, UCSB, p.12. Available at: http://web.physics.ucsb.edu/ fratus/phys103/LN/SR3.pdf

2. Sarah Livesey Dip M.T (2013). Space and Time into a single Continuum. [video] Available at: https://www.youtube.com/watch?v=MO_Q_f1WgQ

3. Sarah Livesey Dip M.T (2013). Space and Time into a single Continuum. [video] Available at:

Published By:

Lattice Science Publication

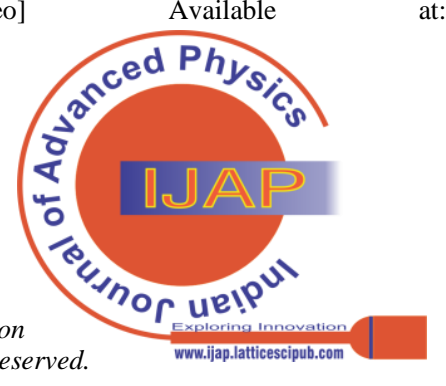


4. Hawkings, S. The Brief History of Time [Book], Chapter 3

\section{AUTHOR'S PROFILE}



Reyan Kumar Sapkota, was a student at St. Xavier's College, Maitighar, Nepal. Reyan has extensively involved himself in research in topics including Physics and Communications. He is involved in various independent and guided research projects Besides Physics, Reyan has been awarded the Young Innovator Award in his country over his engineering endeavors. He has also bagged several awards for his projects. Reyan is also founder and one of the Board of Directors in a student-led organization called Nepal Computer Science Alliance(NECSA). The organization aims to bring every Tech Talents under the same roof and work in various projects to meet a common goal. Overall, Reyan loves Physics and is always involved in making a difference through his endeavors.

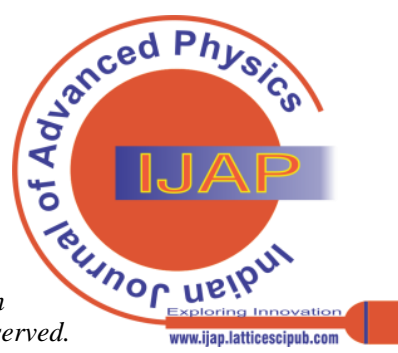

